Recepción: 20 / 04 / 2017

Aceptación: 20 / 05 / 2017

Publicación: 15 / 06 / 2017

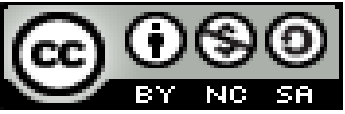

Ciencias de la Educación

Artículo de Investigación

\title{
Influencia de las habilidades cognitivas en el rendimiento académico de los estudiantes de educación general básica
}

\section{Influence of cognitive abilities on the academic performance of basic general education students}

\section{Influência das habilidades cognitivas no desempenho acadêmico dos alunos na educação básica}

\author{
Jéssica E. Posligua-Espinoza ${ }^{\mathrm{I}}$ \\ jessica.posliguae@ug.edu.ec \\ Beatriz G. Vallejo-Vivas II \\ vallejo_beatriz@hotmail.com \\ Erick S. Pazmiño-Peñafiel ${ }^{\text {III }}$ \\ erick.pazminop@ug.edu.ec
}

Correspondencia: jessica.posliguae@ug.edu.ec

I. Economista con Mención en Gestión Empresarial (Especialización Marketing), Magister en Educación Superior, Especialista en Proyectos de Desarrollo Educativos y Sociales, Docente Universidad de Guayaquil, Guayaquil, Ecuador.

II. Magister en Comunicación y Desarrollo; Licenciada en Ciencias de la Comunicación Social; Docente Universidad de Guayaquil, Guayaquil, Ecuador.

III. Magister en Diseño y Gestión de Marca; Licenciado en Diseño y Producción Audiovisual; Universidad de Guayaquil, Guayaquil, Ecuador. 


\section{Resumen}

Las habilidades cognitivas influyen en la calidad del rendimiento académico de los estudiantes, su estudio ha logrado en los estudiantes un mejor desempeño en las tareas educativas, lo que ha traído consigo el análisis para su posterior aplicación en el ambiente educativo.

El contenido del texto está direccionado tanto a docentes como a estudiantes con la determinación de brindar una educación de calidad a los educandos, resolviendo los problemas en las instituciones. Esta investigación, analiza las bases teóricas sobre la importancia del nivel educativo e integral que contribuya al mejoramiento de las habilidades cognitivas del estudiante en su rendimiento académico.

La presente investigación se inicia por un problema bastante común en los estudiantes, la incidencia de las habilidades cognitivas en la calidad del rendimiento académico de los estudiantes, el mismo que se da por diferentes factores, ya sea por ausencia de recursos tecnológicos, metodologías tradicionales, entre otras que no favorecen en nada a la educación si no se las emplean adecuadamente.

El uso de recursos tecnológicos, permitirá difundir el proceso de innovación activando la información en el ambiente educativo, los profesores podrán plasmar sus conocimientos de forma virtual, utilizando herramientas interactivas teniendo la posibilidad de manejar diferentes formatos (texto, imagen fija y en movimiento, con sonido), el objetivo primordial es permitir la realización de actividades interactivas, con animaciones ayudando al direccionamiento de sus contenidos de emprendimiento, presentándolos de forma dinámica en el aula de clases.

Palabras clave: Influencia; Habilidades Cognitivas; Rendimiento Académico. 


\begin{abstract}
The research that follows is intended to seek and take advantage of the benefits provided by technology as a basic tool for third year students of Basic General Education, for this reason it will help develop the best use of the computer as a study medium And pedagogical support. The purpose of this research is focused on technological growth today, this helps to have a specific vision of what is wanted to achieve, which means that it is a reality the contributions of technology or information systems in the Ecuador, for this reason multimedia interactive systems are used much more frequently in the new era of educational globalization that is supported by the use of multimedia tools. In the development of the interactive system the respective didactic strategies are refered, which will promote a significant and motivating learning in the development of each class, deploy abilities and skills for a better learning and thus also to develop with more dynamics the subject to try, this leads to a better understanding of the concepts in Basic General Education students. The purpose of this research is to know the need for teachers to improve each class content. This research aims to help an update of strategies and resources to a globalizing technology that is being developed to improve the teaching process towards students
\end{abstract}

Key words: Technologies, Tools, Basic Education. 


\section{Resumo}

A investigação que se apresenta a seguir, tem a finalidade de busca e aproveitar os benefícios que brinda a tecnologia como a ferramenta básica para os estudantes de terceiro ano de Educação Geral Básica, por esta razão, ajudará a desenvolver o melhor uso do computador como meio de estudo Y apoio pedagógico. O objetivo desta pesquisa, está acionada para o crescimento tecnológico na área de atuação, para ajudar a atingir a sua vida em direto, e assim por diante, é uma realidade das aportaciones da tecnologia dos sistemas de informação em Equador, por esta razão, os sistemas interactivos multimídia são utilizados com muita más frequiência na nova era da globalização educativa que é apoiada com o uso das ferramentas multimídia. No desenvolvimento do sistema interactivo da base de dados e das metodologias, busca-se uma experiência significativa e motivadora em desenvolvimento de cada classe, desplegar capacidades e habilidades para um melhor aprendizado e assim como se desarrolle com mais dinâmica o tema a Tratar, esto conlleva a conseguir uma melhor compreensão dos conceitos em estudantes da Educação Geral Básica. A realização desta pesquisa é como o propósito é a necessidade de ter os documentos para o poder em todo o conteúdo da classe. Esta investigação visa ajudar a uma atualização das estratégias e recursos para uma tecnologia globalizadora que está sendo desenvolvida para melhorar o processo de ensino para os estudantes

Palavras chave: Tecnologias, Ferramentas y Educação Básica 


\section{Introducción.}

Las habilidades y destrezas, están vinculadas como parte de la educación que permite desarrollar conocimientos, como actividades motivadoras que ayuden al desempeño del estudiante aplicando estrategias innovadoras en la asignatura como es la implementación de recursos tecnológicos en el aula de clases. (Araya Ramírez, 2014)

En cuanto a la investigación de campo, se realizó encuestas, dirigidas a los estudiantes de la Educación Básica, al personal docente y director del establecimiento; en la que se reflejan diversos factores que permiten aplicar diferentes métodos en el proceso de enseñanza, entre otros. Se pretende ser un aporte en los estudiantes mediante una propuesta que ayudará a mejorar el rendimiento académico de los estudiantes, cuya finalidad es tener una excelente calidad en el aprendizaje significativo, utilizando un material didáctico innovador como es desarrollo de un software interactivo para ayudar a desarrollar las habilidades cognitivas del estudiante. (Moreira, 2012)

El objetivo de la presente investigación, es contribuir en el ámbito educativo y en el factor de motivación, mejorando considerablemente el proceso enseñanza, ya sea por vía del docente o por el estudiante debido a que ambos están inmersos en este sistema que busca mejorar la calidad de la educación, teniendo como problemática la ausencia de las tecnologías para impartir las clases y además la falta de estrategias que debilitan la educación.

De manera consecuente, la calidad del aprendizaje significativo es importante para poder hablar de la calidad de la educación, por eso es necesario optimizar la estructura cognitiva del educando con nuevas herramientas que la tecnología provee para lograr afianzar conocimientos en 
los estudiantes y que la educación sea más interactiva, participativa y que tenga importancia para el educando. (Orejudo Hernández, 2006)

La investigación está encaminada al uso de la tecnología para impartir las clases de manera interactiva y mejorar el aprendizaje dentro del aula de clases. Los resultados serán reflejados satisfactoriamente en el rendimiento escolar de los estudiantes de Educación Básica.

El bajo rendimiento académico, surge por el hecho de que no se utiliza las herramientas multimedia existentes en el mundo de la tecnología educativa por parte del docente, por tal motivo sus clases son un tanto aburridas y no interactivas y mucho menos motivadoras, por tal razón el rendimiento académico de los estudiantes es bajo. (Enríquez Guerrero, Segura Cardona, \& Tovar Cuevas, 2013)

El docente no tiene acceso a las nuevas herramientas, ya sea por su desconocimiento o por otro factor ajeno a su desempeño, es por eso que esta investigación es de suma importancia; la utilización y aplicación de las nuevas herramientas tecnológicas en los establecimientos educativos de Educación Básica, en la mayoría de casos cuenta con pocos laboratorios de computación por no mencionar que no disponen de laboratorios de informática para un centenar de estudiantes, y para un buen aprendizaje es necesario utilizar un software interactivo, repercutiendo con resultados positivos y a la vez mejorará la calidad de la educación básica en el país, fortaleciendo el conocimiento que ya poseen los estudiantes. (García, Días, Sorte, Díaz Pérez, Rita Leal, \& Gandra, 2014)

El uso de las Tic, ayuda a desarrollar en los estudiantes actitudes creativas e innovadoras para el aprendizaje, además se sentirán inducidos a participar activamente en clases, favoreciendo su desenvolvimiento y la motivación en las diferentes asignaturas. 
La investigación realizada enmarca el software educativo muy útil y aplicable en la presente temática.

La investigación realizada en los estudiantes de Educación Básica, refleja cierto grado de dificultad en el proceso de aprendizaje al no utilizar los nuevos recursos didácticos existentes y que se debe aplicar en los educandos por su influencia en el mundo globalizado del siglo XXI.

Los recursos tecnológicos son de gran importancia y necesarios en la aplicación del sistema educativo para mejorar la calidad de la misma, debido a que proporcionan motivación y actitudes positivas en los estudiantes.

Se realiza la presente investigación para profundizar y conocer con mayor precisión la problemática del rendimiento de los estudiantes, por ello se ha dado pie a esta investigación que tiene el propósito de descubrir cómo incide las habilidades cognitivas en el rendimiento académico de los estudiantes de Educación Básica.

Para la solución de esta temática se efectúa el diseño de una revista virtual como apoyo al proceso de aprendizaje, que permita mejorar el rendimiento académico de los estudiantes, lo cual contribuirá con su aprovechamiento, tanto en el aula como en el laboratorio de informática con el fin de innovar técnicas que se puedan aplicar en la unidad educativa.

El objetivo de esta investigación, es tener docentes capaces de utilizar diversas técnicas educativas innovadoras que refresquen las mentes de los estudiantes. La elaboración de la revista interactiva, contribuirá en que los estudiantes desarrollen de mejor manera sus habilidades cognitivas para que sean capaces de enfrentar retos, que puedan elevar su nivel de aprendizaje, que conciban la idea de investigar, analizar, deducir y asimilar los conocimientos, poniendo en práctica todas sus habilidades, su creatividad y sean capaces de construir sus propios conocimientos. 
Para la ejecución de esta investigación existe toda la apertura y apoyo necesario por parte de las autoridades de las Instituciones de Educación Básica de Guayaquil, los docentes y los padres de familia; donde se desea aplicar de la mejor manera el presente trabajo de investigación, con el fin de lograr los objetivos propuestos en bienestar de los estudiantes, para que estos puedan tener un mejor nivel de aprendizaje.

\section{Materiales y métodos.}

Para el desarrollo del trabajo se emplearon métodos del nivel teórico y del nivel empírico para validar la investigación que se desarrolla desde el punto de vista cualitativo. Se destacan el histórico-lógico, el inductivo-deductivo, la observación, la entrevista, la encuesta, el sistémico estructural y funcional. El enfoque histórico-cultural fue la base del estudio.

\section{Resultados.}

La propuesta de la creación de una revista interactiva, con contenido multimedia, texto, evaluaciones y como adicional, cuenta con un apartado de realidad aumentada para incentivar a los estudiantes a aprender, interactuando y observando, diferentes imágenes creadas por el ordenador, en el software de realidad aumentada. La ejecución de la propuesta está dirigida a los estudiantes de Educación General Básica.

\section{Conclusiones.}

Es necesario que en estos tiempos se promueva el uso de recursos didácticos digitales en el aula de clases, por medio de metodologías dinámicas de innovación, se generaría una nueva forma de pensar altamente necesaria, sobre todo, en épocas encaminadas hacia una cultura tecnológica. 
Se debe considerar al docente como un ente facilitador del proceso de aprendizaje, tiene que asumir los grandes retos de esos cambios que enmarcan a la sociedad actual en el campo de las tecnologías. Y el uso de esta revista virtual cumple con su objetivo de mejorar en el aprendizaje de los estudiantes, de las instituciones de educación general básica.

El docente debe estar en constante renovación, de tal forma, que responda a las necesidades y a las exigencias que demanda la sociedad.

La revolución científico-técnica ha llegado a transformar los procesos educativos, brindando nuevas alternativas para hacer más eficaces los procesos de enseñanza-aprendizaje; se tiene a disposición variedad de recursos didácticos y tecnológicos para revolucionar la educación, por eso resulta un reto para toda persona que debe enseñar y aprender, el hacer uso de esos medios para facilitar el proceso educativo, con el objetivo de romper con el paradigma de sólo utilizar recursos tradicionales como son la pizarra, marcador, borrador y el contenido de la asignatura en textos.

Si el docente emplea estos recursos didácticos digitales como estrategia de innovación dentro del proceso de enseñanza, mejorará el aprendizaje significativo y el desarrollo de las habilidades cognitivas del estudiante.

\section{Bibliografía.}

Araya Ramírez, N. (2014). Las habilidades del pensamiento y el aprendizaje significativo en matemática, de escolares de quinto grado en Costa Rica. Revista Electrónica "Actualidades Investigativas en Educación", 14(2), 1-30.

Enríquez Guerrero, C., Segura Cardona, Á., \& Tovar Cuevas, J. (2013). Factores de riesgo asociados a bajo rendimiento académico en escolares de Bogotá. Investigaciones Andina, 15(26), 654-666.

García, C., Días, P., Sorte, A., Díaz Pérez, J., Rita Leal, A., \& Gandra, M. (2014). El uso de las TIC y herramientas de la Web 2.0 por maestros portugueses de la educación primaria y educación especial: la importancia de las competencias personales. Profesorado. Revista de Currículum y Formación de Profesorado, 18(1), 241-255. 
Jéssica E. Posligua-Espinoza; Beatriz G. Vallejo-Vivas; Erick S. Pazmiño-Peñafiel

Moreira, M. (2012). La Teoría del Aprendizaje Significativo Crítico. Indivisa. Boletin de Estudios e Investigación, 6(1), 83-102.

Orejudo Hernández, S. (2006). Reseña de "CALIDAD DEL APRENDIZAJE UNIVERSITARIO". Revista Interuniversitaria de Formación del Profesorado, 20(3), 327-331. 